\title{
Jet-Tagged Back-Scattering Photons For Quark Gluon Plasma Tomography
}

\author{
Rainer J. Fries ${ }^{\mathrm{a}}$, S. De $\mathrm{e}^{\mathrm{a}, \mathrm{b}}$, D. K. Srivastava ${ }^{\mathrm{b}}$ \\ ${ }^{a}$ Cyclotron Institute and Department of Physics and Astronomy, Texas AEM University, College Station, TX 77845, USA \\ ${ }^{b}$ Variable Energy Cyclotron Centre, 1/AF, Bidhan Nagar, Kolkata - 700064, India
}

\begin{abstract}
Several sources of direct photons are known to contribute to the total photon yield in high energy nuclear collisions. All of these photons carry characteristic and important information on the initial nuclei or the hot and dense fireball created in the collision. We investigate the possibility to separate photons from back-scattering of high momentum quarks off quark gluon plasma from other sources. Their unique kinematics can be utilized through high energy jet triggers on the away-side. We discuss the basic idea and estimate the feasibility of such a measurement at RHIC and LHC.
\end{abstract}

Keywords: Quark Gluon Plasma, Electromagnetic Probes

\section{Introduction}

Photons and dileptons hold great promise as penetrating probes of hot nuclear matter. Their mean free path is short compared to the typical size of the fireball created in high energy nuclear collisions. Hence, once they are created their rescattering can be neglected and they encode valuable information on their creation process. Different sources of direct photons have been discussed in the literature over the years. (i) Hard photons from initial scatterings of partons in the nuclear wave functions. They can be used to extract information about the modification of nucleon wave functions in large nuclei [1]. (ii) Photons fragmenting off jets created in initial hard collisions. Together with the previous source these photons are already present in elementary $p+p$ collisions. Fragmentation photons, besides being sensitive to the same wave function effects as hard initial photons can be suppressed through energy loss of the partons before the photon is radiated off [1]. Hard initial and fragmentation photons can in principle be distinguished experimentally through isolation cuts which check for hadronic activity in a cone around the photon. (iii) Pre-equilibrium photons. This poorly understood photon source is active during the first $\mathrm{fm} / c$ of a nuclear collision when partons are reinteracting but are not thermalized [2]. (iv) Thermal QGP radiation. Thermal emission from an equilibrated quark gluon plasma (QGP) is one of the signature measurements in heavy ion physics [3, 4, 5, 6], as it could serve as proof of deconfinement and as a thermometer for the matter created [7]. Thermal QGP radiation might however not be easily distinguishable from other thermal emission. (v) Thermal radiation from hot hadronic gas. Hadronic emission is an important contribution to the overall yield at low photon momenta [3]. Albeit at lower temperature than the QGP photons the rate does not vary much across the pseudo-critical temperature $T_{c}$ for deconfinement. (vi) Photons from the hadronization processes. Photons from hadronization of QGP - while undoubtedly present [8] - have largely been ignored in the literature so far due to the intrinsically non-perturbative nature of the process. (vii) Photons from

Email address: rjfries@comp.tamu.edu (Rainer J. Fries) 
jets interacting with the medium. It has been argued that jet energy loss in QGP and in hot hadronic matter should be accompanied by photon back-scattering [9, 10] and bremsstrahlung [11]. Back-scattering photons are sensitive to the mean free path of fast partons in QGP and encode information about parton energy loss that is complementary to hadronic measurements. In these proceedings we propose a novel way to measure back-scattering photons from fast partons in QGP.

\section{Back-Scattering Photons}

It is well known in electrodynamics that elastic photon-electron scattering exhibits a sharp peak in backward direction. This Compton back-scattering phenomenon has been used extensively to create collimated beams of high energy photons by scattering an intense laser pulse $\left(E_{\gamma} \sim 1 \mathrm{eV}\right)$ off a beam of high energy electrons $\left(E_{e^{-}} \sim 1-100\right.$ $\mathrm{GeV}$ ) [12, 13]. In quantum chromodynamics the mixed process $q+g \rightarrow q+\gamma$ involves the same type of diagrams at leading order and exhibits the same Compton back-scattering peak. A similar peak can be observed for the annihilation process $q+\bar{q} \rightarrow \gamma+g$. Hence we expect a significant yield of high energy photons from quarks $\left(E_{q} \sim 1-100 \mathrm{GeV}\right)$ scattering off thermal gluons $\left(E_{g} \sim 200 \mathrm{MeV}\right)$ or thermal antiquarks.

In [9] some of us have estimated the rate of high energy Compton back-scattering and annihilation photons from jets interacting with the medium to be

$$
E_{\gamma} \frac{d N}{d^{4} x d^{3} p_{\gamma}}=\frac{\alpha \alpha_{s}}{4 \pi^{2}} \sum_{f=1}^{N_{f}}\left(\frac{e_{f}}{e}\right)^{2}\left[f_{q}\left(\mathbf{p}_{\gamma}, x\right)+f_{\bar{q}}\left(\mathbf{p}_{\gamma}, x\right)\right] T^{2}\left[\ln \frac{3 E_{\gamma}}{\alpha_{s} \pi T}+C\right]
$$

where $C=-1.916 . \alpha$ and $\alpha_{s}$ are the electromagnetic and strong coupling constant, $T$ is the local temperature at $x, f_{q}$ is the phase space distribution of fast quarks interacting with the medium and $e_{f}$ is the electric charge of a quark with the index $f$ running over all active quark flavors. This rate has subsequently also been calculated for virtual photons [14, 15].

We note a few interesting facts about the back-scattering rate. First, it is parametrically proportional to $T^{2} \ln 1 / T$ and hence very sensitive to the medium temperature. Secondly, it is proportional to the quark distribution $f_{q}$ as a function of momentum, position and time. Hence back-scattering photons have a power-law like spectrum that can make a sizable contribution even at intermediate and large photon transverse momentum $P_{T}$. Nevertheless the normalization of this spectrum is still sensitive to the temperature of the medium. This leads to the interesting prospect of finding thermal signatures at large momenta. Furthermore, because the quark distributions evolve with time the back-scattering spectra are sensitive to quark energy loss.

In Refs. [9, 10, 16] it has been noted that jet-medium photons can make a sizable contribution to the total photon yield both at the Relativistic Heavy Ion Collider (RHIC) and the Large Hadron Collider (LHC). They shine particularly bright compared to other sources in the intermediate $P_{T}$ range around $4 \mathrm{GeV} / c$. This comes from the power-law behavior which lifts them above purely thermal sources at those momenta, while on the other hand there is an additional $\sim 1 / P_{T}$ factor [9] that makes the power-law slightly softer than the initial hard photon spectrum which dominates at very large $P_{T}$. However, it is very difficult to experimentally confirm the presence of jet-medium photons, let alone produce a quantitative measurement, by analyzing singe inclusive photon spectra alone. Subsequently it was proposed by some of us to use the azimuthal asymmetry $v_{2}$ around the beam axis as a signature [17]. This is based on the observation that jet-medium photons should have negative $v_{2}$ unlike any of the other photon sources whose $v_{2}$ is positive or vanishing [18]. However, the small absolute size of direct photon $v_{2}$ at intermediate and large momenta has made this impossible with currently available data [16].

Here we propose a novel way to experimentally confirm the existence of jet-medium photons and maybe extract quantitative information from them. The basic idea starts with a classification of hadron-photon or jet-photon correlations inherent in the sources discussed above. It is clear that only initial hard photons, fragmentation photons and jet-medium photons have a significant back-to-back correlation with hadrons or jets. We will focus on jets in the following which lead to cleaner signals and are now routinely measured at LHC and RHIC. Thus by measuring photons on the opposite side of a trigger jet one can eliminate most competing photon sources right away. Next we note that fragmentation photons are concentrated at low $z(\lesssim 0.3)$ where $z=E_{\gamma} / E_{\text {parent jet }}[1,19]$. Hence background from this source can be suppressed by choosing a kinematics which features large $E_{\gamma}$, of the order of the trigger jet energy. 

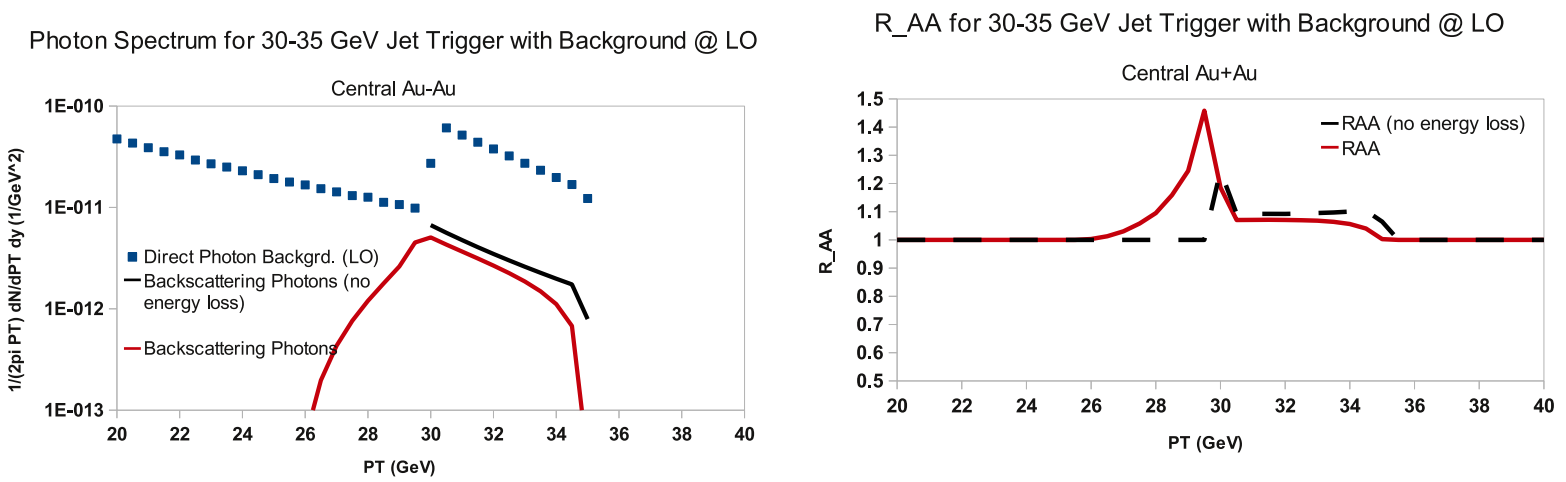

Figure 1: Left panel: photon spectra associated with trigger jets from $30-35 \mathrm{GeV}$ in central Au+Au collisions at RHIC at $\sqrt{s_{N N}}=200 \mathrm{GeV}$. The yield of back-scattering photons with (red solid line) and without (black dashed line) energy loss is compared to the background of initial hard photons and fragmentation photons (blue squares). Right panel: the nuclear modification factor $R_{A A}$ with (red solid line) and without (black dashed line) taking energy loss of leading partons into account.

At leading order (LO) kinematics the transverse momenta of the trigger jet and an associated hard initial photon balance perfectly, $P_{T, \gamma}=P_{T \text {,trigger }}$. Without energy loss of the partons before radiation the back-scattering kinematics would ensure the same momentum correlation with the trigger for back-scattering photons, potentially burying them under the hard direct photon peak. However, if the leading parton of the associated jet suffers from energy loss, the back-scattering photon $P_{T}$ will be shifted away from the trigger $P_{T}$, typically a few GeV below $P_{T \text {,trigger }}$ This shift by itself, if measured, carries valuable information about parton energy loss. At next-to-leading order (NLO) kinematics the back-to-back correlation and the strong momentum correlations get washed out but residual signals remain.

\section{Results}

In this section we present the results of some preliminary studies of the signal (back-scattering photons) and the background (initial hard and fragmentation photons). We use the JETPHOX code [20, 21] for jet-photon and jethadron yields at LO and NLO to calculate the underlying hard process and the background from initial hard and fragmentation photons. The trigger jets were fixed in trigger windows around midrapidity, then the photon spectra were calculated at midrapidity in sectors of $30^{\circ}$ width around the away-side. We use the PPM code [22, 23] to propagate leading jet partons of the away-side jet inside a longitudinally boost-invariant fireball with a $L^{2}$-dependent energy loss (sLPM in [22]) and to convert them to photons according to Eq. (1). The energy loss parameter in PPM is fixed to describe measured single inclusive hadron spectra.

Fig. 1 shows the results for central Au+Au collisions at RHIC $\left(\sqrt{s_{N N}}=200 \mathrm{GeV}\right)$ for a jet trigger window of $E_{T}=30-35 \mathrm{GeV}$ for LO kinematics. Direct hard photons exhibit a distinctive peak inside the trigger window while fragmentation photons slowly rise from the trigger window towards lower $z$. Back-scattering photons without energy loss prior to the Compton or annihilation process are strongly correlated with the trigger window as well. However, if energy loss is switched on they show a distinct shoulder toward smaller momenta due to energy loss of partons before the Compton or annihilation process. We also show the nuclear modification factor $R_{A A}$ which is approximated by the ratio of back-scattering photons (signal) over sum of back-scattering (signal) and hard initial and fragmentation (background) photons. A signal can be seen across the trigger window. A characteristic peak develops once energy loss is switched on. Fig. 2 shows the same for central $\mathrm{Pb}+\mathrm{Pb}$ collisions at the LHC $\left(\sqrt{s_{N N}}=2.76 \mathrm{TeV}\right)$ for a jet trigger window of $E_{T}=100-105 \mathrm{GeV}$. Initial results for backgrounds at NLO accuracy show a decorrelation between trigger and and hard initial photon momentum as expected. This might decrease the signal-to-background by up to a factor two. 

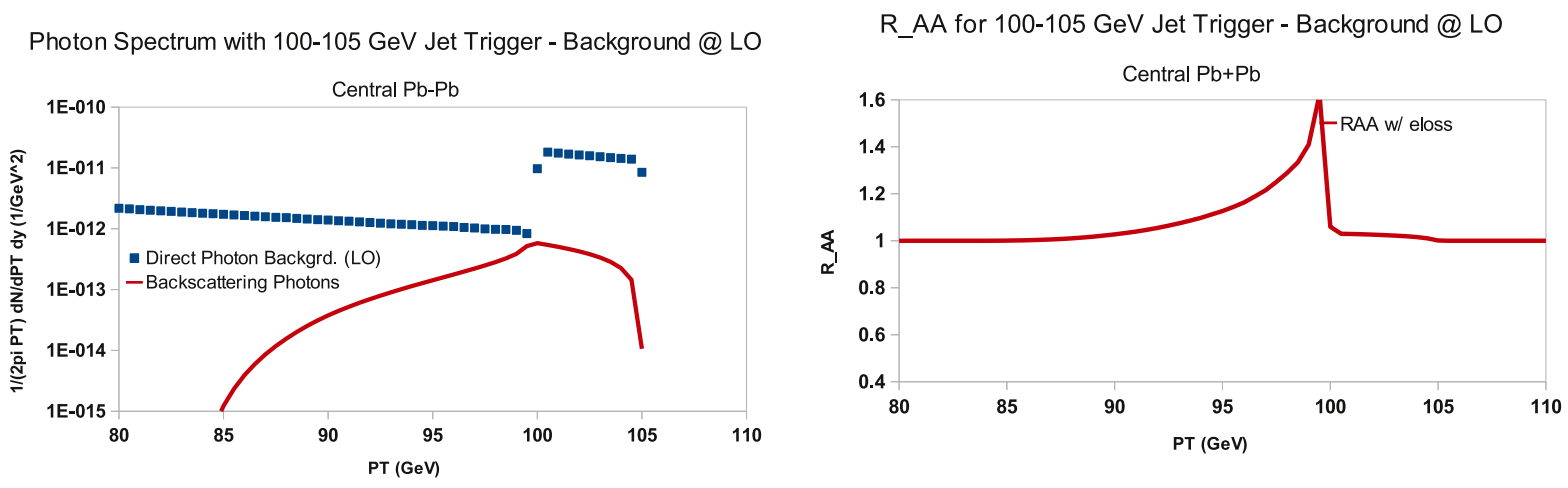

Figure 2: The same as Fig. 1 for $100-105 \mathrm{GeV}$ trigger jets in central $\mathrm{Pb}+\mathrm{Pb}$ collisions at the LHC at $\sqrt{s_{N N}}=2.76 \mathrm{TeV}$. Only the (realistic) case of back-scattering photons with energy loss prior to their emission is shown.

\section{Summary}

Separating and quantitatively using different direct photon sources in high energy nuclear collisions is challenging. We advocate the use of jet triggers to separate back-scattering photons from a background of fragmentation and hard initial photons. We predict that back-scattering photons produce a characteristic enhancement in nuclear modification factors for direct photons on the away side of the trigger, just below the trigger jet energies. This technique completely subtracts all direct photon sources without an underlying hard process. It further suppresses fragmentation photons which amass at low $z$, and hard direct photons which do not suffer from energy loss. The height of the back-scattering peak reflects a quadratic temperature dependence and the shift of the peak down from the trigger window is sensitive to parton energy loss. In the future one should study the effects of trigger jet energy loss which has been neglected so far, as well as add a next-to-leading order calculation of the hard process underlying the back-scattering signal.

This work was supported by NSF CAREER Award PHY-0847538 and by the JET Collaboration and DOE grant DE-FG02-10ER41682.

\section{References}

[1] J. F. Owens, Rev. Mod. Phys. 59, 465 (1987).

[2] S. A. Bass, B. Muller and D. K. Srivastava, Phys. Rev. Lett. 90, 082301 (2003).

[3] J. I. Kapusta, P. Lichard and D. Seibert, Phys. Rev. D 44, 2774 (1991) [Erratum-ibid. D 47, 4171 (1993)].

[4] R. Baier, H. Nakkagawa, A. Niegawa and K. Redlich, Z. Phys. C 53, 433 (1992).

[5] P. Aurenche, F. Gelis, R. Kobes and H. Zaraket, Phys. Rev. D 58, 085003 (1998).

[6] P. B. Arnold, G. D. Moore and L. G. Yaffe, JHEP 0112, 009 (2001).

[7] A. Adare et al. [PHENIX Collaboration], Phys. Rev. Lett. 104, 132301 (2010) |arXiv:0804.4168 [nucl-ex]].

[8] G. Chen, R. J. Fries, in preparation.

[9] R. J. Fries, B. Muller and D. K. Srivastava, Phys. Rev. Lett. 90, 132301 (2003) |nucl-th/0208001].

[10] R. J. Fries, B. Muller and D. K. Srivastava, Phys. Rev. C 72, 041902 (2005) |nucl-th/0507018|.

[11] B. G. Zakharov, JETP Lett. 80, 1 (2004) [Pisma Zh. Eksp. Teor. Fiz. 80, 3 (2004)] |hep-ph/0405101].

[12] R. H. Milburn, Phys. Rev. Lett. 10, 75 (1963).

[13] F. R. Arutyunian and V. A. Tumanian, Phys. Lett. 4, 176 (1963).

[14] D. K. Srivastava, C. Gale and R. J. Fries, Phys. Rev. C 67, 034903 (2003) |nucl-th/0209063|.

[15] S. Turbide, C. Gale, D. K. Srivastava and R. J. Fries, Phys. Rev. C 74, 014903 (2006) |hep-ph/0601042].

[16] S. Turbide, C. Gale, E. Frodermann and U. Heinz, Phys. Rev. C 77, 024909 (2008) [arXiv:0712.0732 [hep-ph]].

[17] S. Turbide, C. Gale and R. J. Fries, Phys. Rev. Lett. 96, 032303 (2006) |hep-ph/0508201|.

[18] R. Chatterjee, E. S. Frodermann, U. W. Heinz and D. K. Srivastava, Phys. Rev. Lett. 96, 202302 (2006) |nucl-th/0511079|.

[19] L. Bourhis, M. Fontannaz, J. P. Guillet and M. Werlen, Eur. Phys. J. C 19, 89 (2001) [hep-ph/0009101].

[20] S. Catani, M. Fontannaz, J. P. Guillet and E. Pilon, JHEP 0205, 028 (2002) |hep-ph/0204023].

[21] P. Aurenche, M. Fontannaz, J. -P. Guillet, E. Pilon and M. Werlen, Phys. Rev. D 73, 094007 (2006) [hep-ph/0602133].

[22] R. Rodriguez, R. J. Fries and E. Ramirez, Phys. Lett. B 693, 108 (2010) |arXiv:1005.3567[nucl-th]].

[23] R. J. Fries and R. Rodriguez, Nucl. Phys. A 855, 424 (2011) |arXiv:1012.3950 [nucl-th]]. 